\title{
POTENCIANDO EL ALCANCE DEL ANÁLISIS PUBLICITARIO CON LA TÉCNICA DE EYE TRACKING: DESARROLLO DE UN SOFTWARE PARA LA EVALUACIÓN DEL IMPACTO PUBLICITARIO
}

\author{
Arbulú, Marcia de la Flor ${ }^{1}$ \\ del Castillo García, Alfredo ${ }^{2}$ \\ Pontificia Universidad Católica del Perú \\ alfredo.delcastillo@pucp.pe
}

Material original autorizado para su primera publicación en la revista académica REDMARKA. Revista Digital de Marketing Aplicado.

https://doi.org/10.17979/redma.2013.01.011.4809

Recibido: 6 Noviembre 2013

Aceptado 2 Diciembre 2013

\section{RESUMEN:}

El avance de las técnicas biométricas en las últimas décadas permite contar con diversas tecnologías al servicio de la investigación de mercados, y específicamente de la evaluación publicitaria, entre las que destacan el Eye Tracking. Este artículo busca destacar la posibilidad de lograr una aplicación y análisis de datos con un mayor valor

\footnotetext{
${ }^{1}$ Licenciada en Psicología con mención en Psicología Social por la Pontificia Universidad Católica del Perú. Áreas de investigación centradas en el Desarrollo Social sobre todo en las influencias de los medios de comunicación y actualmente en el traslado de estos hacia las tecnologías de información y comunicación (TICs), sobre todo en la comprensión de la realidad y el traslado de los procesos de aprendizaje de la lecto-escritura a lo audiovisual. Docente en la Pontificia Universidad Católica del Perú de los cursos de Desarrollo Social Intrapersonal e Interpersonal, Seminario de Tesis en Psicología Social y Prácticas Pre-profesional.

${ }^{2}$ Licenciado en Psicología con mención en Psicología Social por la Pontificia Universidad Católica del Perú. Áreas de interés centradas en: Comportamiento del Consumidor, Metodología de la Investigación, Gestión de la Relación con los Clientes, Segmentación de Mercados y Marketing Relacional. Actual Gerente General de HANDSEARCH SAC y docente en la Pontificia Universidad Católica del Perú del curso de Publicidad e Investigación de Mercados.
}

REDMARKA UIMA-Universidad de A Coruña - CIECID 
agregado en el contexto del uso de las técnicas biométricas para la evaluación publicitaria; en función a la experiencia del desarrollo de un software especializado que genera una capa de análisis de datos adicional a los indicadores de base generados por los sistemas que permiten operar el Eye Tracking.

Palabras clave: seguimiento ocular, evaluación publicitaria, investigación de mercados.

\section{ABSTRACT:}

The advance of biometric techniques in recent decades allows the use of various technologies for market research, specifically for advertisement evaluation, among which the Eye Tracking stands out. This article seeks to highlight the possibility of achieving a better application and data analysis with greater added value in the context of the use of biometric technology for advertising evaluation, according to the experience of the development of a specialized software that generates an additional layer of data analysis generated by the systems that operate the Eye Tracking.

Key words: eye tracking, advertising evaluation, market research.

\section{INTRODUCCIÓN}

En el contexto del significativo desarrollo de nuevas técnicas y metodologías de investigación, en diversos campos de aplicación, como consecuencia del avance 
tecnológico; destaca el crecimiento y sofisticación de las técnicas biométricas, específicamente del Eye Tracking o Seguimiento Ocular.

Esta técnica tiene como uno de sus principales usos, en el marco de su aplicación en la investigación de mercados, permite inferir diversos aspectos relacionados al procesamiento cognitivo por parte del consumidor frente a la publicidad, a través de sus movimientos oculares (Peyrichoux y Robillard-Bastien citado en Tobii Technology, 2009; Tobii Technology, 2010). En este sentido, en función al análisis de los patrones de atención visual del consumidor, se puede identificar qué tanta atención demanda la pieza publicitaria, o qué elementos de la publicidad captan por más tiempo la visualización del consumidor.

Siendo importante destacar que el Eye Tracking es una técnica no intrusiva, que permite registrar lo que el sujeto observa, el intervalo de tiempo de su observación, la trayectoria visual que sigue al observar una publicidad y la dilatación de la pupila ante los estímulos presentados (Bercea, 2011). Operándose este seguimiento visual al identificarse la posición de la cabeza y de las pupilas del sujeto mediante la refracción de un haz de luz infrarroja, emitida por el eye tracker, equipo que opera el Eye Tracking (Nielsen y Pernice, 2009).

No obstante la utilidad del uso de una tecnología como el Eye Tracking en el marco de la evaluación publicitaria -sobre la base de los indicadores y estadísticas que reportan los sistemas que permiten operar estos equipos- se identifica la posibilidad de desarrollar capas de análisis adicionales, en función al desarrollo de algoritmos que trabajan con la data generada por estos sistemas, que le agregan valor a la evaluación de las piezas publicitarias, y cuya automatización evita que este análisis complementario implique una demanda significativa de tiempo de trabajo.

\section{EYE TRACKING}

REDMARKA UIMA-Universidad de A Coruña - CIECID

Año VI, Número 11, (2013), v2 pp. 125-136

http://www.redmarka.org/

ISSN 1852-2300 
La idea básica detrás de la técnica de Eye Tracking es que el movimiento de nuestros ojos puede ser utilizado para realizar inferencias sobre nuestros patrones atencionales y procesamiento cognitivo. Siendo una técnica que se opera por medio de diversos modelos de equipos que reciben la denominación común de eye trackers, los que incorporan la tecnología que permite concretar el seguimiento ocular, es decir, utilizar el reflejo de una luz infrarroja que impacta directamente en el ojo del sujeto, para luego -mediante un avanzado procesamiento de algoritmos y un modelo fisiológico en 3D del ojo- determinar el punto visual exacto del sujeto frente a lo que está observando en un punto específico de tiempo (Tobii Technology, 2009).

Es importante mencionar que para una mayor precisión en la identificación de lo que está observando el sujeto, se requiere de un procedimiento de calibración inicial, en virtud del cual el sujeto observa un punto o conjunto de puntos, mientras que la tecnología de eye tracking registra el valor que corresponde a cada posición de la mirada, con miras a delimitar el marco visual del sujeto (Duchowski, 2007; Nielsen y Pernice, 2009).

La ejecución de la técnica de Eye Tracking implica el análisis de los patrones de atención visual en términos de fijaciones y movimientos sacádicos, términos que hacen referencia a los movimientos típicos de los ojos. La fijación describe la ubicación de la mirada en una posición estática determinada, mientras que los movimientos sacádicos aluden al cambio de una posición a otra (Duchowski, 2007; Zurawicki, 2010). La serie de fijaciones y movimientos sacádicos es denominada scanpath, y es utilizada para el análisis de la percepción visual, cognición, interés y saliencia de los estímulos observados (Bercea, 2011). Es importante mencionar que se obtiene mayor información del análisis de las fijaciones, ya que una mayor atención de los estímulos que se observan implica una mejor reconstrucción visual de los mismos. Infiriéndose que el mundo, y cualquier estímulo específico, es percibido visualmente a través de éstas fijaciones (Tobii Technology, 2010). 
Pueden considerarse cuatro categorías metodológicas en el marco de desarrollo de la técnica del Eye Tracking (Duchowski, 2007):

- En primer lugar se encuentra el electro-oculograma (EOG) que se basa en la medición de las diferencias de potencial eléctrico de la piel, por medio de electrodos que se colocan alrededor del ojo. Esta categoría mide los movimientos oculares en función a la posición de la cabeza.

- En segundo lugar, se encuentran los lentes de contacto especiales, que implican el uso de un objeto de referencia mecánico u óptico montado sobre una lente de contacto que se usa directamente en el ojo (córnea o esclerótica), resultando el método más intrusivo.

- $\quad$ Por otro lado, están las técnicas que utilizan la grabación del movimiento de los ojos en términos de características distinguibles (forma aparente de la pupila, la posición del limbo -el límite entre el iris y la esclerótica-, y los reflejos corneales de una fuente de luz dirigida) agrupadas en foto-oculograma (POG) o videooculograma (VOG).

- $\quad$ Adicionalmente, se encuentran las técnicas basadas en la grabación mediante videos en combinación con la reflexión corneal y pupilar, que utilizan un punto de atención y son utilizadas en la identificación de los elementos en una escena visual, por ejemplo, en aplicaciones interactivas y material gráfico. Su funcionamiento se basa en la utilización de una luz, generalmente infrarroja, que se refleja desde el ojo y es detectada por una cámara de video. La rotación de los ojos brindaría cambios en los reflejos de luz lo cual ayudaría en el análisis de información. Esta categoría de técnicas es la que enmarca la aplicación actual del Eye Tracking, siendo la de uso más extendido, tanto por ser no invasiva como por tener menor costo.

\section{APLICACIÓN DEL EYE TRACKING EN EL ANÁLISIS PUBLICITARIO}

REDMARKA UIMA-Universidad de A Coruña - CIECID

Año VI, Número 11, (2013), v2 pp. 125-136

http://www.redmarka.org/

ISSN 1852-2300 
El uso del Eye Tracking ha permitido lograr un diagnóstico visual efectivo y objetivo de las piezas publicitarias -tanto en soporte audiovisual como gráfico y digital- mediante investigaciones que típicamente implican la presentación de la pieza publicitaria a una muestra de consumidores, registrando la performance visual de la pieza o las piezas presentadas. Siendo muy común el desarrollo de evaluaciones comparativas, sea entre más de una alternativa de la misma pieza publicitaria o evaluando publicidades de distintas marcas o empresas.

Los datos resultantes de estas investigaciones de evaluación publicitaria pueden ser analizados estadística y gráficamente, y proporcionan evidencia de patrones visuales específicos en el marco de la observación de las piezas evaluadas. En ese sentido, mediante la examinación detenida de las fijaciones, movimientos sacádicos, dilatación de las pupilas, parpadeos y una variedad de otros comportamientos, los investigadores pueden determinar mucho sobre la eficacia de una pieza publicitaria (Kalliny y Gentry, 2010).

Uno de los campos de aplicación del Eye Tracking a nivel publicitario son los medios publicitarios y sus piezas, tales como anuncios impresos, on-line o programas patrocinados. Empresas como Kraft Foods, Microsoft, Google, Yahoo, P\&G, IBM, entre otras, la han utilizado en la evaluación de sus elementos de marketing visual (Weder \& Pieters, 2006).

Otra de las aplicaciones del Eye Tracking, cada vez más intensivas, es el campo de la interacción humano computador y en la evaluación de usabilidad (Ghaoui, 2006), específicamente en lo que se refiere a la evaluación de publicidad on-line y, en general, de la interacción con páginas web. El Eye Tracking ofrece la posibilidad de analizar la interacción de los usuarios entre los clics y la cantidad de tiempo que un usuario utiliza entre aquellos clicks. Lo que se refleja en un software encargado de 
recoger los patrones visuales del participante mientras interactúan con los estímulos evaluados. Esto proporciona información valiosa sobre qué características son las más llamativas, cuáles causan confusión y cuáles se ignoran por completo (Duchowski, 2007; Nielsen y Pernice, 2009), pues el movimiento de los ojos, está estrechamente relacionado con la atención visual (Weder \& Pieters, 2006).

De esta manera, en el marco del desarrollo de teorías de atención visual y toma de decisiones basadas en estímulos, el Eye Tracker se presenta como una herramienta capaz de brindar información importante para la evaluación de piezas publicitarias, mediante un método que permite medir objetivamente la atención de los consumidores, ofreciendo como principales ejes diagnósticos respecto a las piezas publicitarias evaluadas:

- dónde fija su vista el consumidor (identificando áreas de interés),

- durante cuánto tiempo se observa cada elemento de la pieza, y

- $\quad$ en qué orden se realiza el barrido visual.

En este contexto de análisis publicitario, la aplicación del Eye Tracking permite identificar, por ejemplo, la proporción de consumidores que se fijan en el logotipo, o en la imagen del producto o en cualquier otro elemento que forme parte de la pieza publicitaria. Además, permite calcular el tiempo transcurrido hasta que el consumidor se fije en cualquier elemento de la publicidad, lo que se traduce en la capacidad de captación de ese elemento de la atención del consumidor, y del alineamiento de este diagnóstico con los objetivos de comunicación previstos para la pieza publicitaria.

Cabe destacar que el Eye Tracking puede utilizarse para la evaluación publicitaria no sólo con carácter de pre-test (antes del lanzamiento de la publicidad) sino también de post-test (tras el lanzamiento de la publicidad). En este último caso, se abren posibilidades para evaluar el impacto a lo largo del tiempo de la publicidad, 
considerando que el nivel de atención e involucramiento por parte del consumidor puede verse afectado por la saturación de información publicitaria reiterativa.

Por ejemplo, en un estudio de la atención visual sobre anuncios impresos -conducido por Rosbergen, Wedel y Pieters (2008)- se aplicó el EyeTracking con el objetivo de analizar el efecto de la exposición repetida a los anuncios impresos. Los autores exploraron el fenómeno de desgaste natural de la publicidad, es decir, el hecho de que los consumidores, disminuyen la atención que le prestan a los anuncios con mayor número de repeticiones (Duchowsky, 2002).

\section{DESARROLLO DE UN SOFTWARE PARA LA EVALUACIÓN DEL IMPACTO PUBLICITARIO}

En el marco de la evaluación de alternativas para el desarrollo de un proyecto de innovación tecnológica -por parte de la empresa de investigación de mercados peruana Handsearch S.A.C. (www.handsearch.net) en conjunto con la Pontificia Universidad Católica del Perú (www.pucp.edu.pe)- se identificó la posibilidad y significativa utilidad de ampliar el alcance y el valor agregado del análisis publicitario por medio de la técnica de Eye Tracking.

Dado que, en el marco del quehacer de la evaluación publicitaria y de los resultados estándar proporcionados por los sistemas que operan el Eye Tracking, se observó que podían desarrollarse capas de análisis adicionales, en función al desarrollo de algoritmos que trabajen con la data generada por estos sistemas. De modo que se obtenga un análisis más profundo y detallado, respecto a las áreas de interés de una pieza publicitaria, con relación a su protagonismo al interior de la pieza, al cálculo de un indicador general de la pieza en función a la performance integrada de cada área de interés o a la fuerza de captación de la atención por parte de una pieza publicitaria, entre otros aspectos relevantes.

REDMARKA UIMA-Universidad de A Coruña - CIECID 
No obstante, se buscaba que esta posibilidad de desarrollo de capas de análisis adicionales sobre la base de la data estándar generada por los sistemas de Eye Tracking, no implicara una demanda adicional significativa de tiempo, siendo el caso que en la ejecución de la investigación de mercados comercial, el tiempo es un bien sumamente escaso. De allí que se definiera como objetivo que el software a desarrollar -que concrete el cálculo de los algoritmos a diseñar- automatice el análisis, evitando que el valor agregado que se buscaba representara un impacto negativo en el presupuesto de las investigaciones a implementar, por demandar una cantidad significativamente mayor de horas de trabajo.

Dados estos antecedentes -e integrados estos objetivos en una propuesta de innovación tecnológica- la empresa de investigación de mercados Handsearch S.A.C. y la Pontificia Universidad Católica del Perú lograron ganar una línea de financiamiento por parte del Programa de Ciencia y Tecnología - FINCyT (www.fincyt.gob.pe), entidad adscrita a la Presidencia del Consejo de Ministros del Estado Peruano, que tiene como misión contribuir al incremento de la competitividad del país, fortaleciendo las capacidades de investigación e innovación tecnológica, y promoviendo la articulación de la Empresa, Universidad y Estado.

Como resultado del proyecto implementado, se desarrolló un software -en plataforma web- que automatiza el cálculo de los siguientes algoritmos diseñados:

- Potencia por área de interés: permite diagnosticar el nivel de potencia, con relación al resto de estímulos, que tiene una determinada área de interés. Para ello debe determinar la relación entre el porcentaje de personas que vieron una determinada área de interés de la pieza vs. el tiempo total de visualización de la misma.

- Protagonismo del área de interés: permite identificar si determinada área de interés de la pieza publicitaria funciona como un elemento relevante o como un

REDMARKA UIMA-Universidad de A Coruña - CIECID 
distractor, construyendo una matriz de 4 cuadrantes, determinados tanto por el tiempo promedio de la primera fijación como por la duración de la misma.

- Atracción del área de interés: permite calcular el nivel de atracción inicial y, por tanto, el poder de captación de la atención del consumidor de cada área de interés de la pieza publicitaria.

- Saliencia perceptual del área de interés: busca encontrar la relación entre el tiempo promedio de visualización de un área de interés y el tiempo transcurrido para la primera fijación en esta área de interés.

- Efectividad del área de interés: busca encontrar la relación entre el tiempo de visualización de determinada área de interés y el espacio que ésta área de interés ocupa respecto al total de la pieza publicitaria.

- $\quad$ Puntaje visual general: permite determinar un puntaje visual general por pieza publicitaria, de modo que su performance visual pueda ser comparada con otras piezas. Con tal objetivo, se obtiene la potencia promedio de todas las áreas de interés que conforman una pieza publicitaria, para calcular una potencia visual general por cada publicidad evaluada.

- $\quad$ Puntaje visual general ponderado: permite determinar un puntaje visual general por pieza publicitaria, de modo que su performance visual pueda ser comparada con otras piezas; pero incluyendo -a diferencia del algoritmo anterior- un factor ponderador de cada área de interés, en función a su importancia estratégica en el marco de los objetivos de comunicación previstos.

Adicionalmente a la configuración del cálculo de estos algoritmos -en el marco del desarrollo del software- se logró cumplir el objetivo referido a que este análisis no implique la demanda de una cantidad significativamente mayor de horas de trabajo. Mediante un análisis de tiempos y movimientos, se encontró que el software desarrollado permite calcular los algoritmos diseñados en un tiempo 120 veces menor al que se necesitaría para realizar su cálculo manual. Sumándose el hecho relevante 
del menor margen de error asociado a este cálculo automatizado y verificado de los algoritmos, en comparación a las diversas fuentes de error potencial relacionadas a un cálculo manual de los mismos.

El presente artículo -en el contexto del software diseñado y, en general, del proyecto implementado- busca promover la innovación tecnológica, especialmente en nuestros países latinoamericanos, mediante el desarrollo de sistemas y aplicativos que permitan un análisis de datos con un mayor valor agregado en el marco del uso de técnicas biométricas para la evaluación publicitaria. Apareciendo como técnicas potenciales para este tipo de desarrollo: la actividad electrodérmica, la frecuencia cardíaca, la pupilometría y el reconocimiento facial, entre otras.

\section{Referencias:}

Bercea, M. (2011) Anatomy of methodologies for measuring consumer behavior in neuromarketing research. University of Iaşi, Romania. Romanian National Authority.

Ghaoui, C. (2006) Encyclopedia of Human Computer Interaction- Eye Tracking in $\mathrm{HCl}$ and Usability Research, pp 211-219, 2006.

Duchowski, A (2002) A breadth-first survey of eye-tracking applications. Behavior Research Methods, Instruments, \& Computers, 34 (4), 455-470.

Duchowski, A. (2007) Eye Tracking Methodology: Theory and Practice. Springer: 2nd edition.

Jacob, R (s.f.) Eye Tracking in Advanced Interface Design. Human-Computer Interaction Lab Naval Research Laboratory. Washington, D.C.

REDMARKA UIMA-Universidad de A Coruña - CIECID 
Kalliny, M. \& Gentry, L. (2010) Marketing in the 22nd Century: A look at four promising concepts. Asian Journal of Marketing, 4 (3), pp. 94 - 105.

Wedel, M., \& Pieters, R. (2008). Eye tracking for visual marketing. Foundations and Trends in Marketing, 1(4), 231-320

Nielsen, J. \& Pernice, K. (2009) Eyetracking Web Usability. New Riders Press.

Pool, A \& Ball, L (2006) Eye Tracking in Human-Computer Interaction and Usability Research: Current Status and Future Prospects. Encyclopedia of HumanComputer Interaction. Ed. C. Ghaoui, Idea Group, Inc. Pennsylvania, pp. 211- 219.

Tobbi Techonology (s.f.) Tobii Eye Tracker Research, recuperado el 16 de octubre de 2013, de http://www.tobii.com/en/eye-tracking-research.

Tobbi Techonology (2009) Retrospective Think Aloud and Eye Tracking. Comparing the value of different cues when using the retrospective think aloud method in web usability testing. Whitepaper by Tobii Technology. September, 2009.

Tobbi Techonology (2010) An introduction to eye tracking and Tobii Eye Trackers. Whitepaper by Tobii Technology. January 27, 2010.

Zurawicki, L. (2010) Neuromarketing, Exploring the Brain of the Consumer. Springer-Verlag Berlin Heidelberg, pp. 42-53.

Zhiwei Zhu \& Qiang Ji (2004) Eye and gaze tracking for interactive graphic display. Machine Vision and Applications (2004) 15: 139-148. 